\title{
IS-LM: uma história?
}

\author{
IS-LM: a bistory?
}

ALEXANDRE AUGUSTO SEIJAS DE ANDRADE* MATHEUS ALBERGARIA DE MAGALHÃES**;***

\begin{abstract}
RESUMO: Para alguns economistas, o modelo IS-LM já passou, correspondendo a uma fase remota da história do pensamento econômico. Neste artigo, tentamos demonstrar que isso não é necessariamente verdade. Ao mesmo tempo, aproveitamos a longevidade do modelo para fornecer uma breve descrição de alguns eventos em Macroeconomia. Além disso, chamamos a atenção para as crescentes diferenças entre macroeconomia "acadêmica" e "prática", fato que pode indicar a existência de problemas relativos ao ensino e às aplicações no campo. Apesar de todas as falhas do modelo, acreditamos que ele ainda não entrou no salão de ícones históricos da Economia e ainda pode ser útil, pelo menos no sentido de treinar novas gerações de alunos com conceitos macroeconômicos básicos.

PALAVRAS-CHAVE: Macroeconomia; economia Keynesiana; modelo IS-LM.
\end{abstract}

ABSTRACT: For some economists, the IS-LM model is already past, corresponding to a remote phase of the history of economic thought. In this paper, we try to demonstrate that this is not necessarily true. At the same time, we take advantage of the model's longevity to provide a brief account of some events in Macroeconomics. Also, we call attention for the increasing differences between "academic" and "practical" macroeconomists, a fact that may indicate the existence of problems pertaining to teaching and applications in the field. Despite all the model's flaws, we believe that it hasn't entered to the hall of historical icons of Economics yet and it may still be useful, at least in the sense of training younger generations of students with basic macroeconomic concepts.

KEYWORDS: Macroeconomics; Keynesian economics; IS-LM model.

JEL Classification: A2; A22; A23; B22; E1; E12.

\footnotetext{
* Instituto de Pesquisas Econômicas da Universidade de São Paulo - IPE-USP, São Paulo/SP, Brasil. E-mail: aaandra@usp.br.

* Instituto de Pesquisas Econômicas da Universidade de São Paulo - IPE-USP, São Paulo/SP, Brasil. E-mail: matt@usp.br. Submetido: janeiro 2003; aceito: novembro 2003.

$* *$ Os autores agradecem os comentários detalhados e as sugestões de Ana Maria Bianchi, Arthur Barrionuevo Filho, Cláudia Heller, Gilberto Tadeu Lima, João Hildebrando Bocchi, Maria Angélica Borges, Raul Cristóvão dos Santos, Willy de Góes e de um parecerista anônimo desta revista, bem como a alguns dos participantes do VIII Encontro Nacional de Economia Política. O segundo autor agradece ainda o apoio financeiro do Sasakawa Young Leader Foundation Fellowship (SYLFF), embora valha a ressalva de que as opiniões aqui contidas não refletem, de modo algum, as visões dessa instituição ou de algum de seus membros. Também vale a ressalva de que os erros e idiossincrasias que forem perpetuados ao longo da história devem-se única e exclusivamente aos autores.
} 


\section{INTRODUÇÃO}

"IS-LM survived because it proved to be a marvellously simple and useful way to organize and process some of the main macroeconomic facts."

Robert M. Solow, Mr. Hicks and the classics.
“(...) if Hicks hadn't invented IS-LM in 1937, we would end up inventing it all over again.”

Paul Krugman, There's something about macro.

Já se passaram mais de sessenta anos desde a publicação do artigo "Mr. Keynes and the classics: a suggested interpretation”, de autoria de John R. Hicks (1937). Neste artigo, o autor descrevia, de maneira simplificada, um sistema de equações relacionando os mercados de bens, monetário, e o de ativos, ao mesmo tempo em que procurava resumir tais idéias em termos geométricos, a partir de gráficos com duas curvas por ele denominadas de "SI" (que representava o equilíbrio no mercado de bens ou, equivalentemente, a igualdade entre poupança e investimento) e "LL" (que representava o equilíbrio no mercado monetário ou a igualdade entre oferta e demanda de moeda). Na verdade, apesar de Hicks procurar realizar uma abordagem simplificada do tema, seu objetivo básico não era em nada modesto. Baseado em um trabalho anterior, Hicks procurava, em última instância, sintetizar as principais idéias contidas no livro (então recém-publicado) A Teoria Geral do Emprego, do Juro e da Moeda, de John Maynard Keynes (1936).

Talvez, na época, Hicks não pudesse ter idéia do impacto de seu artigo: além do esquema por ele proposto estar presente na maioria dos livros-texto de Macroeconomia da atualidade, tal esquema tornou-se, em certo momento, um instrumental largamente utilizado na análise de políticas econômicas alternativas e seus efeitos sobre o nível de renda.

$\mathrm{Na}$ verdade, hoje em dia, a última afirmação não tem um caráter plenamente consensual. Em primeiro lugar, embora tal esquema esteja, de fato, contido na grande maioria dos livros-texto de macroeconomia, já existem livros onde é feita uma abordagem distinta da área; o que reflete, no mínimo, o fato de que não há um consenso atual sobre o uso desse esquema como recurso didático.

Para comprovar essa última afirmação, construímos uma lista de alguns livrostexto de Macroeconomia, procurando verificar quais deles continham pelo menos uma seção dedicada ao modelo IS-LM. Na tabela 1, abaixo, expomos os resultados desse empreendimento. Nosso critério de seleção dos capítulos dos livros mencionados foi o seguinte: citamos o primeiro capítulo/seção de cada livro onde fosse feita alguma menção ao modelo IS-LM. Caso contrário, colocamos o termo n.c. ("nada consta") no espaço conveniente. 
Tabela 1: Lista de livros-texto usados em cursos

de Economia onde aparece o modelo IS-LM

\begin{tabular}{lc}
\hline \multicolumn{1}{c}{ Autores } & Capítulo/Seção com IS-LM \\
\hline Hall e Taylor (1989) & Graduação \\
Barro (1990) & Cap. 4 \\
Dornbusch e Fischer (1991) & Cap. 20 \\
Gordon (1993) & Cap. 4 \\
Mankiw (1995) & Cap. 3 \\
Sachs e Larrain (1995) & Cap. 9 \\
Blanchard (2001) & Cap. 12 \\
\hline & Cap. 5 \\
\hline Blanchard e Fischer (1989) & Pós-graduação \\
Sargent (1987a) & Cap. 10 \\
Sargent (1987b) & Cap. 2 \\
Romer (1996) & n.c. \\
Ljungqvist e Sargent (2000) & Cap. 5 \\
\hline
\end{tabular}

Fonte: elaboração própria.

Podemos notar a partir da inspeção da tabela acima que todos os livros-texto utilizados em cursos de graduação de nossa amostra citam ou expõem brevemente o modelo IS-LM. $1^{1} \mathrm{O}$ mesmo não é verdade, no entanto, em termos de livros-texto de pós-graduação que se dedicam, em geral, a tópicos relacionados à fronteira de conhecimento em Economia. Assim, enquanto Sargent (1987a, cap. 2) chega a dedicar um capítulo inteiro ao modelo (como uma extensão do modelo de seu primeiro capítulo, o modelo "clássico"); Blanchard e Fischer (1989, cap. 10, seção 10.4) e Romer (1996, cap. 5, seção 5.2) dedicam meramente algumas seções ao tema. Em livros-texto de pós-graduação como Sargent (1987b) e Ljungqvist e Sargent (2000), não encontramos nenhuma menção ao modelo. Em suma, poderíamos constatar, a partir da tabela acima, que o modelo IS-LM vem, de fato, per-

\footnotetext{
${ }^{1}$ Embora essa intensidade possa variar de acordo com a obra consultada. Assim, enquanto livros como Dornbusch e Fischer (1991) baseiam a maior parte de sua exposição no modelo IS-LM, Barro (1990) elabora uma análise nitidamente distinta, baseada em microfundamentos e na hipótese de marketclearing, com o modelo IS-LM sendo descrito apenas na parte final do livro. Colander (2003) também apresenta alguma evidência bibliométrica relacionada ao tema, chegando à conclusão de que o modelo IS-LM vem aparecendo cada vez mais em artigos de caráter pedagógico e relacionados à história do pensamento econômico. Evidência semelhante à nossa está contida em Vercelli (1999, p. 207). Ver ainda Policano (1985).
} 
dendo prestígio como recurso didático em cursos de pós-graduação, embora o mesmo não ocorra, em geral, no que concerne à graduação.

Apesar desse esquema ainda ser visto como extremamente útil em termos de análises aplicadas, o mesmo não pode ser dito em termos estritamente acadêmicos. Assim, há autores que chamam atenção para a crescente diferença entre economistas "práticos" e "acadêmicos" e as conseqüências decorrentes dessa crescente divisão (Tobin, 1986; Blinder, 1988; Mankiw, 1990). Ainda assim, em ocasião recente, alguns macroeconomistas renomados, quando indagados a respeito da existência de um core macroeconômico, responderam com um vigoroso "sim" a tal indagação e, ao descreverem os elementos básicos desse core, incluíram o modelo IS-LM como um de seus principais elementos. ${ }^{2}$

Apesar das críticas ao modelo IS-LM ao longo das últimas décadas, recentemente foram até propostas modificações em sua estrutura básica, de modo que este passasse a refletir de forma mais acurada a conduta dos bancos centrais modernos (Romer, 2000). Na verdade, alguns bancos centrais, hoje em dia, valem-se de modelos macroeconométricos constituídos por diversos setores representando a economia, cuja inspiração básica vem de uma estrutura semelhante à do modelo IS-LM. ${ }^{3}$

Um historiador do pensamento macroeconômico do século XX poderia notar que o modelo IS-LM apresentou, ao longo desse período, um desenvolvimento como esquema analítico que ocorreu de modo paralelo aos principais eventos que vinham ocorrendo na própria Macroeconomia como disciplina.

Assim, tal esquema foi, gradualmente, adquirindo importância crescente na disciplina, com seu apogeu ocorrendo ao longo da "era keynesiana" nos anos 1960 nos Estados Unidos, para começar a sofrer um processo de declínio a partir de meados da década de 1970, quando da crítica (teórica e empírica) de Lucas e associados aos modelos keynesianos e monetaristas da época. A década de 1980, por sua vez, parecia uma época de extrema divisão no ramo. De um lado, os economistas ligados à abordagem novo-clássica propunham maior rigor em termos analíticos, valendo-se, para tanto, de modelos dinâmicos de equilíbrio geral. Do outro, economistas que ainda admitiam ter algum vínculo com idéias "keynesianas" — não tão em voga na época - realizavam um esforço de pesquisa ligado à construção de uma base teórica mais sólida para essas idéias. ${ }^{4}$

\footnotetext{
${ }^{2}$ Ver, a esse respeito, os artigos de Blanchard (1997), Blinder (1997), Solow (1997) e Taylor (1997) do simpósio da edição de maio de 1997 da American Economic Review sobre a existência de um core em Macroeconomia. Vale aqui a ressalva de que pode ter ocorrido, na ocasião, um certo viés de seleção da amostra de macroeconomistas considerados, uma vez que o único economista selecionado para tal simpósio, além dos citados acima, foi Martin Eichenbaum.

${ }^{3}$ No caso brasileiro, ver, por exemplo, o trabalho recente de Alves e Muinhos (2003), onde os autores constroem um modelo macroeconométrico de médio porte para o país, bem como as referências lá contidas.

${ }^{4}$ Esse debate é exposto de forma mais detalhada em Magalhães (2000), onde estão contidas diversas referências relacionadas a esse estágio recente da história do pensamento macroeconômico. Ver ainda
} 
À primeira vista, pode parecer que um esforço de pesquisa no sentido de contextualizar alguns dos acontecimentos ocorridos em Macroeconomia usando o modelo IS-LM como primeira aproximação deveria ser realizado por algum macroeconomista maduro o suficiente para ter acompanhado alguns dos debates ocorridos ao longo do tempo, mas que, ao mesmo tempo, conseguisse manter uma certa distância e imparcialidade em relação ao tema. Tal impressão é provavelmente verdadeira. Mesmo assim, como jovens economistas com um certo interesse em Macroeconomia, não podemos deixar de ficar intrigados com as crescentes diferenças existentes entre "práticos" e "acadêmicos", conforme ressaltado por alguns dos autores citados acima.

Por outro lado, acreditamos que exatamente devido ao fato de não termos acompanhado a maior parte da evolução da Macroeconomia como disciplina, talvez possamos ter um certo grau de distanciamento do tema, o que pode ser algo bastante útil a nossos propósitos. De qualquer forma, cabe avisar aos eventuais leitores, que nosso objetivo aqui é bastante modesto. Não pretendemos, de modo algum, realizar uma extensa resenha sobre a evolução do modelo IS-LM (ou da Macroeconomia moderna, em última instância). Também não estamos preocupados em enfatizar a legitimidade desse modelo em relação à Teoria Geral. Isso já foi feito por outros que nos antecederam. ${ }^{5}$ Do mesmo modo, leitores que esperarem encontrar aqui alguma nova derivação ou insight relacionado ao modelo IS-LM - à Romer (2000), por exemplo - provavelmente ficarão decepcionados.

Há cerca de dez anos, Carlos Magno Lopes (Lopes, 1992a) organizou, na Revista Brasileira de Economia, um simpósio sobre as contribuições de Hicks, ondediversos economistas expunham pontos de vista distintos relacionados à obra intelectual desse autor. Recentemente, no ano de 2003, foi realizada uma conferência na Duke University, organizada por Michel De Vroey e Kevin Hoover, intitulada "The IS/LM model: its rise, fall, and strange persistence". A chamada de trabalhos da conferência contém uma série de questões interessantes relacionadas ao modelo. O mesmo é válido em relação às tentativas de resposta oferecidas por alguns dos participantes do evento, conforme é o caso, por exemplo, das contribuições de Backhouse e Laidler (2003), Bordo e Schwartz (2003) e Colander (2003). Esses acontecimentos demonstram, no mínimo, a atualidade do tema do presente trabalho. ${ }^{6}$

Woodford (1999) e Blanchard (2000), que equivalem a referências que cobrem um período de tempo mais amplo do que o primeiro autor citado.

${ }^{5}$ Ver, a esse respeito, Darity e Young (1995), por exemplo, que realizam uma resenha sobre a evolução histórica desse modelo, com especial ênfase nos esforços de formalização ocorridos posteriormente à publicação da Teoria Geral.

${ }^{6}$ Após a preparação da versão preliminar do presente artigo, tomamos conhecimento de outras referências que confirmam nossas preocupações básicas. Exemplos nesse sentido são os trabalhos recentes de Vercelli (1999) e Heller (2002) que indagam, basicamente, a respeito das razões relacionadas à longevidade prolongada do modelo IS-LM. Como coloca essa última autora: “(...) a literatura econômica é rica no que diz respeito ao debate em torno do grau de lealdade com que o modelo IS-LM representa a Teoria Geral, 
$\mathrm{Na}$ verdade, queremos apenas chamar atenção para alguns pontos relacionados ao tema que nos parecem relevantes. Esperamos fornecer um número de perguntas significativamente superior em relação ao número de possíveis respostas. Nosso artigo tem como título uma frase interrogativa, já que nosso questionamento básico refere-se ao fato de o modelo IS-LM ter se tornado um ícone da história do pensamento econômico, ou não. Nossa intenção é, de certa forma, semelhante à de Easterly (1999). No caso específico desse trabalho, é ressaltado o fato de que, apesar de o modelo Harrod-Domar ser tido como obsoleto em termos acadêmicos, ele continua sendo usado extensivamente em órgãos como o FMI e o Banco Mundial. De forma similar a esse autor, atentamos para uma situação semelhante que ocorre no caso do modelo IS-LM; em especial, para as crescentes diferenças entre o que é ensinado nos cursos de Macroeconomia e como tal conhecimento é aplicado, de fato, na prática. Se este artigo conseguir cumprir o objetivo de propiciar uma certa reflexão a respeito da evolução da Macroeconomia como disciplina, e até mesmo puder iniciar um debate a esse respeito, tanto melhor.

Assim sendo, queremos, em última instância, propiciar ao leitor uma breve visão panorâmica da evolução da Macroeconomia desde meados da década de 1930 até os dias de hoje, utilizando o modelo IS-LM como proxy para tal panorâmica, e, ao mesmo tempo, questionar a respeito de uma certa mudança metodológica ocorrida no ramo, principalmente a partir da década de $1970 .^{7}$ Adicionalmente, há aqui uma preocupação em termos didáticos. Nesse sentido, estamos preocupados basicamente com a seguinte questão: o modelo IS-LM pode ser tido como uma ferramenta útil no ensino de Macroeconomia ainda hoje?

Nosso artigo está dividido da seguinte maneira: primeiramente, descrevemos o processo de criação desse modelo, que equivaleu, na verdade, a uma descoberta (quase) simultânea de três economistas. Em seguida, é descrito o ambiente de extrema confiança e otimismo na capacidade analítica desse modelo, vigente nos Estados Unidos ao longo das décadas de 1950 e 1960; enquanto, na seção seguinte é descrito um ambiente diametralmente oposto, em que o modelo passou a ser alvo de intensa crítica e reformulação ao longo da década de 1970, caindo praticamente em desuso nas duas décadas seguintes (pelo menos, no meio acadêmico norteamericano). $\mathrm{Na}$ outra seção, discutimos a diferença entre economistas "práticos" e "acadêmicos" e as pro-

mas é surpreendentemente pobre no que diz respeito às explicações do sucesso do modelo." (Heller 2002, p. 1). Ambos os trabalhos possuem, em certos aspectos, um maior grau de profundidade do que o nosso, bem como amplas listas de referências bibliográficas relacionadas ao tema.

\footnotetext{
${ }^{7}$ Uma abordagem histórica da Macroeconomia como disciplina que se vale do uso do modelo IS-LM como proxy para tal empreendimento não poderia estar, de certa forma, dissociada da evolução do modelo DA-OA (demanda e oferta agregadas), dada a conexão próxima existente entre esses dois esquemas analíticos. Ainda assim, a título de simplificação, no caso presente, restringimos nossa análise ao modelo IS-LM apenas. Agradecemos a Gilberto Tadeu Lima por ter chamado nossa atenção para esse ponto.
} 
váveis causas dessa crescente divisão; ao mesmo tempo em que tratamos do uso didático do modelo. Finalmente, são apresentadas as conclusões do artigo.

\section{UMA DESCOBERTA (QUASE) SIMULTÂNEA}

O mais influente dos primeiros artigos que propunham atribuir uma estrutura matemática à Teoria Geral de Keynes é o artigo de Hicks "Mr. Keynes and the Classics: a suggested interpretation" (1937). Este artigo foi um dos três apresentados em uma sessão do Encontro da Sociedade de Econometria, realizado em Oxford, em setembro de 1936, em um simpósio sobre a Teoria Geral. De fato, esta foi uma sessão memorável, pois, além de Hicks, os dois outros autores a apresentar seus trabalhos foram James Meade e Roy Harrod. De acordo com Darity e Young (1995, p. 7), Hicks teria tido acesso aos dois outros artigos antes de preparar o seu. Parece haver indícios também de que ele teria lido um outro artigo, de autoria de David Champernowne (1936), que procurava destacar as diferenças entre as economias "clássica" e "keynesiana". Champernowne submeteu o seu trabalho ao Review of Economic Studies. Como Hicks era editor do referido periódico, é muito provável que ele também o tenha lido antes de escrever o seu artigo.

Hicks era professor da London School of Economics (LSE) desde 1925. Sua linha de pesquisa era Economia do Trabalho, e ele já havia, inclusive, publicado alguns trabalhos na área. Durante sua estada na LSE, Hicks foi designado por Lionel Robbins para ocupar-se das cadeiras de Equilíbrio Geral e Risco, em virtude de sua sólida formação matemática (Franco, 1983, p. 128). Some-se a este fato as leituras que Hicks fez dos artigos de Meade, Harrod e Champernowne e tem-se o famoso artigo de 1937. De fato, a influência proveniente da leitura desses três artigos foi maior do que a proveniente de Walras. O modelo de Meade (1937) tinha ao todo dez equações, sendo que sete eram idênticas às do modelo apresentado por Hicks (1937). Já o artigo de Harrod (1937) centrava-se na distinção entre as economias "clássica" e "keynesiana".

Estes foram os elementos que condicionaram a leitura que Hicks fez da Teoria Geral. É diretamente deles que o autor deriva o diagrama IS-LM. De acordo com Hicks (1980, pp. 141-2): "the idea of the IS-LM diagram came to me as a result of the work I had been doing on three-way exchange, conceived in a walrasian manner". Nas palavras de Franco (1983, p. 130):

“Os elementos principais da Teoria Geral (...) podiam ser entendidos como hipóteses específicas, às quais deveria ser acrescentada a do salário nominal fixo, de um modelo de equilíbrio geral walrasiano. As equações independentes deste sistema walrasiano, que determinavam os valores em equilíbrio geral para a renda agregada e a taxa de juros, formavam as curvas IS-LM para o 'caso especial' keynesiano (....).” 
O artigo de Hicks (1937) é estruturado da seguinte maneira: na primeira seção, o autor retrata o modelo clássico, de forma bem simples, onde a renda agregada é determinada pela quantidade de moeda em circulação. A igualdade entre poupança e investimento a um certo valor da taxa de juros, determina a divisão da produção entre bens de consumo e bens de investimento. Na segunda seção do artigo, é caracterizada a economia de Keynes, em que Hicks tenta identificar as descobertas daquele autor. Em um primeiro momento, Hicks destaca a dificuldade da teoria clássica em determinar a renda, a não ser pelo fator monetário. Em seguida, enfatiza a importância da hipótese de preferência pela liquidez, entendida como a sensibilidade da demanda por moeda à taxa de juros. Desta, supondo-se uma oferta fixa de meios de pagamento, dependeria o funcionamento do multiplicador. No final dessa seção, Hicks caracteriza o "caso especial” keynesiano como uma situação na qual a taxa de juros atinge um valor mínimo e a expansão monetária é então incapaz de elevar o nível de renda. Na terceira seção do artigo, Hicks considera tanto a economia "clássica" quanto a "keynesiana" como casos específicos de uma teoria mais ampla, denominada por ele de "Teoria Geral Generalizada".

Para cada modelo, o autor apresentou três equações, representando o mercado de bens, o setor de investimento e o mercado monetário. A partir daí, a abordagem básica de sua estratégia de comparação foi integrar as referidas equações em duas curvas, SI (que caracterizaria o equilíbrio no mercado de bens) e $L L$ (correspondente ao equilíbrio no mercado monetário), hoje em dia conhecidas como IS e $L M{ }^{8}$

Essa maneira de interpretar o sistema keynesiano obscureceu, de fato, diversos pontos essenciais da Teoria Geral, como tem sido insistentemente repetido por diversos economistas pós-keynesianos. ${ }^{9}$ Apesar disso, durante um longo período, o modelo IS-LM chegou a ser visto como a mais famosa e influente interpretação de Keynes (Franco, 1983, p. 125). Ironicamente, o próprio Hicks, a quem é atribuída a criação do modelo, confessou em uma entrevista, que nunca chegou a considerar o modelo como uma interpretação plenamente fidedigna da Teoria Geral, conforme atesta o trecho abaixo:

"Even at my most keynesian time, well, as I said, I never thought that the whole General Theory could be done in one diagram. I myself

\footnotetext{
${ }^{8}$ Darity e Young $(1995$, p. 6) especulam que tal mudança de denominação deva-se, em última instância, a esforços oriundos de Alvin Hansen, um dos maiores divulgadores do modelo nos Estados Unidos. Além de seus trabalhos sobre economia keynesiana, o trabalho de Modigliani (1944) também foi fundamental no sentido de "popularizar" o modelo IS-LM na academia norte-americana da época.

${ }^{9}$ Em certa ocasião, Joan Robinson chegou a rotular alguns economistas keynesianos (de inclinação neoclássica) de "keynesianos bastardos". Ver, a esse respeito, Harcourt (1987). Backhouse e Laidler (2003), por sua vez, argumentam que tanto o modelo IS-LM, como a própria Teoria Geral, acabaram colocando de lado diversas idéias e insights importantes da literatura sobre Economia Monetária e Ciclos de Negócios do período entre-guerras. Ver ainda Bianchi (1992), que trata de aspectos metodológicos da obra de Hicks, com especial ênfase ao caráter revolucionário "invisível” desta.
} 
certainly did not want to confine myself to that diagram. There were a good many more things that I wanted to say, even at that stage." (Hicks apud Klamer, 1989, p. 177).

Em um artigo escrito para a conferência "50 anos depois de IS-LM", Patinkin (1990) considera que é preciso tentar responder a duas perguntas ao considerar as críticas a que o modelo geralmente é submetido: a primeira diz respeito ao fato de o esquema ser ou não uma interpretação válida da Teoria Geral; a segunda, se o modelo representa uma construção analítica válida e útil. A resposta do autor para ambas perguntas é afirmativa. Ao contrário de King (2000), por exemplo, que propõe uma nova estrutura para o modelo ao apontar várias limitações do mesmo, como, por exemplo, a ausência de expectativas racionais e mudanças no nível de preços, Patinkin afirma que é preciso levar em conta o contexto no qual o livro de Keynes foi escrito, qual seja, o período posterior à Grande Depressão. O tema central da Teoria Geral, para o autor, não era o nível de preços, mas sim o desemprego. É possível notar uma visão otimista em Patinkin (1990) até mesmo quando o autor comenta o artigo de Hicks de 1981. Hicks não estaria afirmando ser o instrumental proposto por ele uma interpretação inválida da Teoria Geral, mas apenas explicitando as limitações do modelo, de um ponto de vista mais rigoroso. Patinkin deduz que Hicks estaria querendo ressaltar a necessidade de se tratar com maior rigor e cuidado a análise do tempo na ciência econômica. Mas isso, de maneira alguma, muda a resposta das duas perguntas que Patinkin se propôs a responder inicialmente.

$\mathrm{Na}$ verdade, em certa altura de sua carreira, Hicks chegou a mudar de inclinação teórica, fazendo uma profunda reavaliação pessoal de seu trabalho anterior. No final da década de 1970, ele escreveu um artigo (Hicks, 1981), no qual aponta algumas falhas existentes no modelo IS-LM. Nesse artigo, o autor reconhece que as duas curvas não poderiam ser colocadas no mesmo diagrama, pois uma refere-se a uma relação de fluxo, e a outra, de estoque. Mas isso já é outra história. ${ }^{10}$

\section{AUGE DURANTE UMA ERA KEYNESIANA}

Apesar de ter sido inicialmente concebido na Inglaterra, o modelo IS-LM acabou tendo sua consolidação plena ocorrendo nos Estados Unidos. A nova geração de macroeconomistas das décadas de 1950 e 1960 acabou usando o modelo extensivamente tanto em nível acadêmico quanto prático.

\footnotetext{
${ }^{10}$ Sobre o processo de reavaliação pessoal de Hicks, ver Hicks (1980), Lima (1992, pp. 41-43), Lopes (1992b) e a entrevista contida em Klamer (1989). Críticas adicionais ao modelo IS-LM estão contidas em Leijonhufvud (1987). Os artigos de Solow (1984) e Patinkin (1990), por sua vez, correspondem a defesas em relação a algumas das principais críticas de Hicks ao modelo.
} 
Paul Samuelson chegou a chamar a atenção para o surgimento de uma "Síntese Neoclássica" que, segundo ele, poderia ser definida da seguinte maneira:

"In recent years 90 percent of american economists have stopped being 'keynesian economists' or 'anti-keynesian economists'. Instead they have worked toward a synthesis of whatever is valuable in older economics and in modern theories of income determination. The result might be called neo-classical economics and is accepted in its broad outlines by all but about 5 per cent of extreme left wing and right wing writers." (Samuelson 1955, p. 212 apud Lima, 1992, p. 136) ${ }^{11}$

Nessa mesma época, passaram a ocorrer esforços no sentido de fornecer maior validade empírica a essa abordagem. Assim, assistiu-se, ao longo do período, à construção de modelos macroeconométricos de grande porte. Na verdade, tais modelos equivaliam a complexos sistemas de equações simultâneas, com o mais conhecido desses modelos sendo o modelo MPS (MIT-Penn-Social Research), construído em parceria por pesquisadores dessas instituições (MIT, Universidade da Pensilvânia [EUA] e o Social Research Council). ${ }^{12}$ Esses modelos estavam diretamente baseados no modelo IS-LM, contendo seus elementos básicos: um setor financeiro, um setor de investimento e um setor relacionado a decisões de consumo/ estoques (Goodfriend e King, 1997, p. 234).

Havia um enorme clima de confiança nas ferramentas então disponíveis, o que apenas confirmava a existência, na prática, de um "consenso keynesiano", conforme sugerido na definição da Síntese Neoclássica de Samuelson. As discussões de política econômica da época podiam ser feitas basicamente em termos do modelo IS-LM - muitas vezes acrescido de alguma versão da curva de Phillips, responsável por representar o lado da oferta agregada -, com as questões mais importantes do debate dizendo respeito ao valor de alguns parâmetros das curvas básicas que constituíam esse modelo.

Nesse período, o modelo IS-LM parecia definitivamente fazer parte da ortodoxia econômica vigente, tanto em termos acadêmicos (incluindo-se aí seu uso como recurso didático) quanto aplicados (já que era extensivamente usado por economistas de agências governamentais). Ainda assim, tamanha popularidade não poderia durar tanto tempo, conforme acabou sendo comprovado pelos eventos da década seguinte, descritos na próxima seção.

\footnotetext{
${ }^{11}$ Sobre a Síntese Neoclássica, ver ainda a breve resenha contida em Blanchard (1987).

12 O modelo MPS original tinha cerca de sessenta equações comportamentais. Com o tempo, foram feitos incrementos adicionais nesse modelo, chegando-se a mais de duzentas equações. Para um resumo da evolução histórica de modelos macroeconométricos usados no FED, ver Brayton et al. (1997).
} 


\section{CRÍTICA E DESCRENÇA}

A década de 1970 assistiu a uma série de eventos que iriam mudar, de forma permanente, o modo de se pensar em Macroeconomia. Os dois choques do petróleo, ocorridos ao longo dessa década, bem como a alta das taxas de juros nos Estados Unidos, ocasionaram um cenário que acabou abalando profundamente o consenso intelectual da "era keynesiana".

No meio acadêmico, alguns economistas passaram a questionar resultados advindos de modelos keynesianos. Embora tais modelos já tivessem sido criticados anteriormente, a natureza das novas críticas parecia distinta. O debate "keynesianos versus monetaristas", ocorrido na década anterior, estava, na verdade, inserido em uma estrutura comum de análise, com as discussões da época ocorrendo basicamente em termos da magnitude de alguns parâmetros do modelo IS-LM. O modelo IS-LM, aliás, representava essa estrutura comum de análise, conforme citado na seção anterior. ${ }^{13}$

No caso da década de 1970, o debate parece ter alcançado outro patamar. Os novos-clássicos questionavam hipóteses básicas de modelos keynesianos, procurando ressaltar suas principais inconsistências, bem como as conseqüências (desastrosas, segundo eles) de se continuar usando tais modelos para a condução da política econômica. ${ }^{14}$ Provavelmente, uma das críticas mais conhecidas do gênero foi aquela contida em Lucas e Sargent (1979). Embora nesse artigo os autores tenham certa preocupação em expor algumas das idéias ligadas à sua abordagem, seu objetivo básico era demonstrar a possibilidade de mudanças de política econômica alterarem parâmetros estruturais de modelos macroeconométricos, afetando, em última instância, parâmetros das formas reduzidas estimadas de maneira complexa; o que já era conhecido há algum tempo como a "crítica econométrica de Lucas” (Lucas, 1976).

Em relação ao modelo IS-LM, as críticas eram de duas naturezas: uma empírica e outra teórica. O primeiro tipo de crítica, na verdade, relacionava-se a esse modelo de forma indireta, uma vez que equivalia à crítica econométrica de Lucas, estando dire-

\footnotetext{
13 Tal afirmação pode ser comprovada a partir do fato de que tal debate pode ser apresentado, em termos didáticos, através do modelo IS-LM, via os casos "clássico" e "keynesiano" ("armadilha de liquidez") (ver Dornbusch e Fischer, 1991, p. 164). Entretanto, vale aqui a ressalva de que os principais expoentes do Monetarismo da época possuíam fortes reservas em relação ao modelo IS-LM. Friedman, por exemplo, só usou esse modelo em uma única ocasião na década de 1970, para tentar estabelecer um meio comum de comunicação com seus oponentes, embora tenha se arrependido desse empreendimento posteriormente. Brunner e Meltzer, por sua vez, apesar de criticarem o modelo, acabaram elaborando uma versão mais refinada do mesmo. Uma visão histórica detalhada da posição monetarista pode ser encontrada no trabalho recente de Bordo e Schwartz (2003).

${ }^{14}$ Vale notar que os modelos keynesianos já vinham sendo criticados por outros economistas também, além de novos-clássicos e monetaristas, como era o caso de críticas advindas de autores pós-keynesianos e marxistas. Para uma contextualização histórica de todo esse processo crítico, pelo menos até a década de 1980, ver Klamer (1988, pp. 1-26).
} 
cionada a modelos macroeconométricos como o MPS, que eram fundamentados, em última instância, no modelo IS-LM. O segundo tipo de crítica dizia respeito à ausência de microfundamentos no modelo IS-LM, em especial, a um tratamento inadequado das expectativas no modelo (muitas vezes, tidas como constantes).

Aparentemente, a macroeconomia novo-clássica parecia ter atingido seu objetivo (explícito) de derrubar o Consenso Keynesiano e se tornar a nova ortodoxia vigente na década de $1970 .{ }^{15}$ Como conseqüência direta desses acontecimentos, o modelo IS-LM parecia então ter sido banido das discussões acadêmicas da época, passando a haver, inclusive, uma certa conotação negativa associada ao rótulo "keynesiano".

Os anos 1980, por sua vez, assistiram a um debate entre duas novas correntes de pensamento macroeconômico, cuja origem estava, de certa forma, associada à macroeconomia novo-clássica.

De um lado, havia economistas que, baseados nas recomendações metodológicas contidas em Lucas (1980), procuravam verificar as implicações lógicas de se conduzir uma análise rigidamente baseada em microfundamentos e mercados equilibrados. Tal esforço de pesquisa estava basicamente ligado ao que posteriormente convencionou-se denominar de "modelos de ciclos reais de negócios" (RBC; "realbusiness-cycle models"). ${ }^{16}$

De outro lado, estavam aqueles economistas preocupados em fundamentar alguns insights keynesianos - o que não fora feito antes pelos membros da Síntese, sendo motivo de intensas críticas -, ao mesmo tempo em que buscavam fazê-lo sob o novo framework de análise proposto pelos novo-clássicos. Para tanto, também partiam de modelos em que havia agentes com expectativas racionais e mercados em equilíbrio. As diferenças ficavam por conta das hipóteses adicionais nesses modelos. No caso, hipóteses como a de concorrência imperfeita em alguns mercados poderia evitar que preços ou salários fossem plenamente flexíveis nos mesmos, por exemplo, gerando resultados distintos de modelos novo-clássicos. Esta corrente ficou conhecida como "novo-keynesiana". ${ }^{17}$

1515 Autores como Tobin (1986) e Blinder (1988) discordam dessa afirmativa, procurando qualificar sob que condições foi possível ocorrer uma certa dominância da macroeconomia novo-clássica. Blinder (1988, pp. 278, 285), por exemplo, coloca que, além de tal dominância só ter ocorrido na academia, ela praticamente se restringiu apenas aos Estados Unidos; sendo, em última instância, resultado de uma combinação entre um certo "avanço metodológico" (em termos de ferramental analítico) e um "retrocesso ideológico". Ambos os autores citados acreditam que a macroeconomia novo-clássica apresentou fracassos empíricos consideráveis.

${ }^{16}$ Uma extensa resenha relacionada a modelos deste tipo está contida em Stadler (1994). Cooley (1995) equivale a uma coletânea contendo alguns avanços recentes relacionados a essa agenda de pesquisa.

17 Gordon (1990) equivale a uma resenha relacionada a essa abordagem. Ver ainda os volumes editados por Mankiw e Romer (1991a, b), contendo algumas das principais contribuições do gênero. Não parece haver um consenso pleno acerca de pontos comuns a essa abordagem. Mankiw (1992), por exemplo, enumera uma série de pontos relacionados a ela, embora admita que tal lista possa variar de acordo com o economista keynesiano indagado, chegando a colocar que os economistas pertencentes a esse 
A essa altura, o modelo IS-LM parecia amplamente esquecido na academia norte-americana, embora alguns novo-keynesianos advogassem que sua análise era plenamente compatível com esse modelo (ver, por exemplo, Mankiw, 1990, p. 1648). Em um simpósio sobre economia keynesiana contemporânea, King (1993) indaga se a macroeconomia novo-keynesiana seria capaz de "ressuscitar" o modelo IS-LM (como diz o próprio título de seu artigo). Esse autor aponta algumas limitações do modelo IS-LM, em especial, a sua incapacidade de incorporar a questão das expectativas de maneira adequada. Para tanto, procura ver as implicações da incorporação da hipótese de expectativas racionais sobre o lado da demanda agregada.

Para King, o conceito de expectativas racionais desafia a lógica do modelo ISLM de forma fundamental. Modelos que incluem expectativas racionais destroem tais separações: primeiramente, porque expectativas sobre o futuro exigem que o longo e o curto prazos sejam considerados em conjunto; segundo, porque expectativas relacionam, ao mesmo tempo, os setores monetário e de poupança. $\mathrm{O}$ autor acaba concluindo que, a insistência no uso do modelo IS-LM contribui para que se postergue o desenvolvimento de uma nova geração de modelos macroeconométricos, nos quais expectativas racionais desempenham um papel central na análise. O autor conclui seu artigo com duas recomendações básicas:

"First, economists should be (...) constructing new small-scale dynamic theoretical models to take the place of the IS-LM apparaturs. The framework will be the basic neoclassical model; rational expectations will play a central role; and the effects of various frictions will be systematically explored. Second, economists should be (...) constructing medium-size dynamic models that can serve as laboratories for thinking about the details of policy choice. Ultimately, that activity is best done as a joint product by researchers working in academic and policy institutions.” (King, 1993, p. 80)

Tais recomendações estão, na verdade, diretamente relacionadas a uma proposta que viria a ser advogada por esse autor em uma ocasião posterior, qual seja, o surgimento de uma nova Síntese Neoclássica (NSN) (Goodfriend e King, 1997). Essa versão moderna da Síntese compartilharia o mesmo espírito que a original, no sentido de incorporar elementos "clássicos" e "keynesianos" na análise das flutuações econômicas. No caso, a nova Síntese poderia ser definida por dois elementos básicos, segundo os autores:

(i) a incorporação de expectativas racionais e otimização intertemporal em modelos macroeconômicos dinâmicos;

grupo poderiam ser conhecidos como "novos monetaristas", dada a existência de uma certa afinidade com as idéias de Friedman. Blanchard (1992), por sua vez, parece lamentar a ausência de uma maior coesão em termos de contribuições do grupo. 
(ii) a incorporação de concorrência imperfeita e um processo de ajuste lento dos preços (Goodfriend e King, 1997, p. 255).

Ou seja, a NSN equivaleria, na verdade, a uma síntese entre modelos RBC e novo-keynesianos. No caso, a parcela "RBC" da Síntese apareceria em termos da metodologia empregada (via construção de modelos de equilíbrio geral dinâmico); enquanto a parcela "keynesiana" surgiria em termos da incorporação de "fricções" (e.g., preços e salários rígidos) nesses modelos. ${ }^{18}$ Esse tipo de abordagem parece bastante promissor e já vem sendo extensivamente usado em Macroeconomia no caso de diversas questões, inclusive àquelas relacionadas aos efeitos reais da política monetária, por exemplo. ${ }^{19}$

Por outro lado, há esforços recentes que procuram refinar o modelo IS-LM. Romer (2000) é, no caso, o primeiro exemplo que vem à mente quando se fala disso. O raciocínio básico do autor é relativamente simples: o modelo IS-LM, tal como originalmente concebido, não parece mais adequado em termos didáticos ou de análise de políticas econômicas, devendo, portanto, ser reformulado. Em termos de política econômica, o autor parte do fato observado de que os bancos centrais, em geral, quando da condução da política monetária, levam em consideração, principalmente, as taxas de juros como variável de análise, ao invés da oferta monetária, conforme suposto originalmente em diversas versões do modelo. Assim, o autor propõe um modelo distinto, o modelo IS-MP-IA, onde os termos MP e IA servem para designar "política monetária" ("monetary policy”) e "ajuste da inflação" ("inflation adjustment"), respectivamente, embora não esteja preocupado em fornecer um modelo rigidamente fundamentado. Seu interesse básico, no caso, parece estar relacionado a fins didáticos e práticos, conforme citado acima. Para o autor, o fato de o modelo IS-LM não possuir microfundamentos pode ser visto, na verdade, como uma vantagem, uma vez que, mesmo os modelos microfundamentados mais simples parecem bem mais complicados - em termos analíticos - do que o modelo IS-LM (Romer, 2000, pp. 152-3).

Um outro artigo recente, que também apresenta uma nova estrutura ao mo-

\footnotetext{
${ }^{18}$ Danthine (1997) coloca que uma das principais contribuições de modelos RBC ocorreu em termos metodológicos e que um provável "sucessor" do modelo IS-LM como workhorse básico em Macroeconomia teria de atender a um número maior de critérios básicos do que esse último modelo originalmente havia atendido, ao mesmo tempo em que mantivesse um compromisso entre performance e simplicidade.

${ }^{19}$ Uma aplicação interessante nesse sentido é o modelo proposto por McCallum e Nelson (1997), em que esses autores derivam as curvas IS e LM a partir de um modelo de otimização dinâmica com moeda na função-utilidade (modelo Brock-Sidrausky). Em termos didáticos, vale a pena citar a abordagem de Mankiw (1995, cap. 14), que realiza uma introdução de modelos RBC a partir de uma versão modificada do modelo IS-LM e do esquema DA-OA. Estas duas aplicações demonstram, de certa forma, o porquê da longevidade do modelo, já que este é um modelo "plástico" nas palavras de Heller (2002, p. 1), com ampla capacidade de adaptação a mudanças no ambiente econômico, conforme colocado adicionalmente por Vercelli (1999). Infelizmente, isto representa, ao mesmo tempo, uma deficiência básica do modelo, já que ele é ambíguo e, em última instância, virtualmente não-falseável (Vercelli, 1999, p. 215).
} 
delo IS-LM, é o de King (2000). O autor é um pouco mais rigoroso do que Romer (2000) ao introduzir expectativas racionais e endogeneizar o nível de preços, explicitando novas equações para o modelo. A principal diferença em relação ao modelo original de Hicks (1937) é a de que o nível de preços é um fenômeno monetário. Essa idéia pode ser representada de duas formas: primeiro, as equações do modelo não podem ser resolvidas para todas variáveis endógenas sem a especificação de uma regra de condução da política monetária; segundo, o nível de preços responde a mudanças no estoque nominal de moeda tanto no curto, quanto no longo prazo. As expectativas de inflação são incorporadas tanto na equação de Fisher, quanto na curva de Phillips. Além disso, na nova curva IS, a renda do período presente depende também da expectativa da renda no período posterior.

Em uma tentativa de verificar empiricamente a validade do modelo IS-LM, Galí (1992) estima um modelo estrutural de vetores auto-regressivos para a economia norte-americana, procurando verificar se as propriedades dinâmicas do modelo estimado são condizentes com as previsões advindas do modelo teórico. Seus resultados tendem, em grande parte, a dar o suporte empírico necessário ao modelo ISLM e, em última instância, a teorias keynesianas, em geral. É feita a ressalva, entretanto, de que uma fração considerável das flutuações no produto norte-americano deve-se também a choques de oferta, o que tende a enfraquecer, de certa forma, o resultado básico do artigo. De qualquer forma, esse tipo de abordagem parece bastante interessante, pelo menos no sentido de legitimar parcialmente o uso do modelo. Uma extensão natural, no caso, seria realizar estudos empíricos desse gênero que considerassem uma ampla amostra de países (além dos Estados Unidos), bem como englobassem diversos intervalos de tempo distintos, como forma de confirmar as conclusões de Galí (1992) de uma maneira mais genérica.

\section{5. "PRÁTICOS" VERSUS "ACADÊMICOS" E A DIDÁTICA NO ENSINO DE MACROECONOMIA}

Conforme citado na introdução do artigo, é possível notar, ao longo das últimas duas ou três décadas, um processo de crescente diferenciação entre macroeconomistas "práticos" e "acadêmicos", um fato já ressaltado anteriormente por outros economistas (Tobin, 1986; Blinder, 1988; Mankiw, 1990). Ou seja, de um lado estão macroeconomistas "acadêmicos", preocupados, em geral, com um maior rigor formal em termos analíticos; ${ }^{20}$ enquanto, de outro, estão os macroeconomistas "práticos" que, apesar de não serem tão rigorosos nos termos citados, valem-

\footnotetext{
${ }^{20}$ Embora não queiramos com isto insinuar, de modo algum, que não haja uma preocupação por parte desses economistas em explicar a realidade que os cerca.
} 
-se de técnicas que lhes parecem úteis a problemas aplicados, tendo, de certa forma, uma postura mais pragmática do que os primeiros.

$\mathrm{Na}$ verdade, dado o crescente processo de especialização ocorrido no ramo, é natural que ocorram essas diferenças. O que parece estranho, no entanto, é que tais diferenças venham aumentando tanto nos últimos anos. É provável que, hoje em dia, a maior parte dos economistas de instituições como o Banco Mundial e o FMI não entendam completamente palestras proferidas por economistas teóricos que sejam expoentes em alguma área avançada da Macroeconomia. Por outro lado, é provável que platéias acadêmicas em certas universidades não apreciem plenamente trabalhos de economistas das instituições citadas.

Talvez isto esteja relacionado à tão conhecida falta de consenso em Macroeconomia, bem como à existência de toda uma série de incentivos no meio acadêmico em direção a um maior grau de diversificação e especialização da produção científica. ${ }^{21}$ Isto fica evidente não apenas pelas colocações dos economistas acima citados, mas, principalmente, pela diferença - em termos de ferramental - entre esses dois tipos de economistas. Por exemplo, Brayton et al. (1997), ao descreverem a evolução do uso de modelos macroeconométricos no Federal Reserve System, colocam que:

"Large-scale macro models are by their nature slow to evolve. This is both a blessing and a curse. Changes in models respond to shifts in the consensus view, as opposed to that of the latest cutting-edge research. This helps avoid the pitfall of constantly changing policy advice and interpretation. On the other hand, models can easily become out of date and irrelevant." (Brayton et al., 1997, p. 76)

Ou seja, apesar de desenvolvimentos teóricos levarem a avanços na construção de modelos econométricos, tal correspondência não é exatamente perfeita, o que se deve ao fato de que, além de alguns dos modelos mais recentes exigirem substanciais recursos computacionais em termos de estimação, nunca é claro, em um dado instante do tempo, se um certo insight teórico se revelará importante o suficiente a ponto de ser implementado em análises cotidianas de política econômica.

De qualquer forma, alguns macroeconomistas chegam a ver esse crescente processo de divisão como algo positivo, como é o caso, por exemplo, de Mankiw (1990) que coloca que técnicas recentes, tidas como avançadas hoje em dia, podem ser úteis ao estudo da Macroeconomia no futuro. Danthine (1997, p. 143), por sua vez, indaga-se a respeito da possibilidade do uso de técnicas sofisticadas tornar-se

\footnotetext{
${ }^{21}$ Uma evidência empírica relacionada à falta de consenso em Economia equivale ao estudo de Frey et al. (1984), realizado para cinco países selecionados. Sobre a tendência de especialização crescente em Economia, ver Woodford (1999, p. 31).
} 
algo cada vez mais natural na disciplina, conforme já ocorre em outras áreas (como Finanças, por exemplo).

Em termos didáticos, o modelo IS-LM certamente não possui mais o prestígio que teve em cursos de graduação há cerca de vinte anos, devido às inúmeras críticas que sofreu ao longo de todo esse período, conforme citado acima. Ainda assim, fica uma questão básica a esse respeito: o modelo IS-LM não pode ser útil no sentido de familiarizar o aluno com noções básicas de Macroeconomia? A resposta a essa questão parece ser afirmativa, conforme atesta Lawrence Christiano, um célebre macroeconomista contemporâneo, em entrevista recente a Carlos Uzabiaga (Uzabiaga, 1998):

"I think that the IS-LM model is a very useful language for talking about macroeconomics with undergraduates. All the ideas of economics that have been discussed in the past couple of decades can be articulated with this framework, with minimal setup costs for the student. (...) The IS-LM model is a language. Its strength is its extreme flexibility, it can accommodate different elements, you can use it to express almost any idea. That's an advantage for undergraduate teaching, it may also be an advantage when thinking casually. But, it is probably not appropriate for research.” (Uzabiaga, 1998, p. 186)

Por outro lado, em termos de pesquisa, a resposta também parece ser afirmativa, pelo menos de acordo com Olivier Blanchard:

"Can macroeconomists use small models such as the IS-LM model or the Taylor model (...)? Or should they use only fully specified dynamic general equilibrium models, now that such models can be solved numerically? Put in these terms, it is obvious that this is an incorrectly framed debate. Intuition is often obtained by playing with small models. Large explicit models then allow for further checking and refining. Small models then allow conveying of the essence of the argument to others. At this stage, I believe that small models are indeed underused and undertaught. Small, back-of-the-envelope, models are much too useful to disappear, and I expect that methodological divide will also fade away." (Blanchard, 2000, p. 1405)

Até mesmo Lucas, em seu Methods and Problems in Business Cycle Theory (Lucas, 1980) reconhece o valor do modelo IS-LM, pelo menos como uma abstração útil à teorização em Macroeconomia:

"There is, certainly, much of interest in the General Theory that is not captured either in Hicks's diagram or Modigliani's equation system (...). While there is some truth (...) in this view, it misses what I believe to 
be the more essential truth (...) that progress in economic thinking means getting better and better abstract, analogue economic models, not better verbal observations about the world. (...) To extract from the General Theory a simple graphical method for thinking about national income determination is not, I believe, to vulgarize its contribution. Vulgarity in economics would more appropriately be defined as criticizing or caricaturing an abstract (and hence potentially useful) model because it leaves something out." (Lucas, 1980, p. 700)

Krugman (1999b, p. 1), por sua vez, realiza uma defesa enfática do modelo em termos de seu potencial de aplicações práticas:

"Now you might say, if this stuff is so out of fashion, shouldn't it be dropped from the curriculum? But the funny thing is that while old-fashioned macro has increasingly been pushed out of graduate progra$\mathrm{ms}$ - it takes up only a few pages in either the Blanchard-Fischer or Romer textbooks that I am assigning, and none at all in many other tracts - out there in the real world it continues to be the main basis for serious discussion. After 25 years of rational expectations, equilibrium business cycles, growth and new growth, and so on, when the talk turns to Greenspan's next move, or the prospects for EMU, or the risks to the Brazilian rescue plan, it is always informed - explicitly or implicitly by something not too different from the old-fashioned macro (...). Why does the old-fashioned stuff persist in this way? I don't think the answer is intellectual conservatism. Economists, in fact, are in general neophiles, always looking for something radical and different. Anyway, I have seen over and over again how young economists, trained to regard IS-LM and all that with contempt if they even know what it is, find themselves turning to it after a few years in Washington or New York. There's something about primeval macro that pulls us back to it (...)."

Ironicamente, esses economistas citados construíram suas carreiras seguindo direções opostas. Ao passo que Blanchard e Krugman se consideram economistas de inclinação keynesiana, Christiano dedicou um considerável esforço de pesquisa à construção de modelos de equilíbrio geral dinâmico, em geral ligados à abordagem RBC. Lucas, por sua vez, é um dos fundadores da macroeconomia novo-clássica. O fato de economistas de vertentes diametralmente opostas terem opiniões semelhantes pode ser um indicativo de que o modelo IS-LM é não apenas um poderoso recurso didático, dada a sua simplicidade, mas também um modelo útil em aplicações práticas e, até mesmo, em termos acadêmicos (embora essa última afirmação seja questionável). Restará saber no futuro se tal impressão é, de fato, verdadeira. 
Neste artigo procuramos expor, de forma sucinta, a evolução do modelo ISLM ao longo do tempo, desde sua origem, em meados da década de 1930. Esperamos, a partir de tal exposição, chamar atenção para alguns acontecimentos recentes em Macroeconomia, tanto em termos de substância quanto de sociologia da profissão.

Apesar das inúmeras críticas (e as conseqüentes transformações) sofridas por esse modelo, acreditamos em sua utilidade para dois fins básicos: primeiro, didático; segundo, de política econômica.

Em termos didáticos, o modelo pode ser útil exatamente devido à sua extrema simplicidade analítica, o que pode facilitar de modo significativo o aprendizado de conceitos básicos em Macroeconomia por estudantes de graduação. Já alcançada uma certa maturidade na disciplina, é natural que os alunos sejam instruídos em técnicas e modelos mais sofisticados; embora, é claro, possa haver uma certa discussão acerca do grau ideal desse tipo de instrução. Concordamos, por outro lado, que pode ser muito mais difícil para o aluno médio familiarizar-se com modelos mais rigorosos (com microfundamentos, por exemplo); mas que, uma vez incorporados conceitos dessa natureza, o aluno pode ter uma maior facilidade na transição em direção a métodos mais refinados e, em última instância, a cursos de pós-graduação; ao contrário do que vem ocorrendo atualmente. ${ }^{22}$

Em termos de política econômica, reconhecemos a crescente divisão entre macroeconomistas "práticos" e "acadêmicos". Ainda assim, acreditamos que a incorporação de alguns avanços teóricos em questões aplicadas pode levar bastante tempo, o que torna plenamente compreensível o fato de macroeconomistas "práticos" continuarem utilizando modelos/técnicas tidos como "ultrapassados" por macroeconomistas "acadêmicos". De qualquer forma, reparando as diversas ocasiões em que Paul Krugman (um economista "acadêmico" com forte interesse em questões aplicadas) alertou para a necessidade de se valorizar modelos simples sem se preocupar tanto com o rigor analítico (Krugman, 1999a, b), não podemos deixar de achar que um bom insight deva ser valorizado (e aplicado), mesmo quando não houver fundamentos teóricos suficientes para subsidiar tal empreitada intelectual, na mesma linha de argumentação de Summers (1991). Quem sabe, assim, este artigo não terá representado apenas uma breve história do modelo IS-LM, para passar a ser, de fato, um relato parcial da evolução desse esquema analítico.

\footnotetext{
22 A destoância existente entre cursos de graduação e pós-graduação em Economia equivale a um fenômeno que ocorre no exterior, conforme atesta o estudo de Colander e Klamer (1990).
} 


\section{REFERÊNCIAS BIBLIOGRÁFICAS}

ALVES, Sergio L.A. e MUINHOS, Marcelo K. (2003). Medium-size macroeconomic model for the Brazilian economy. Banco Central do Brasil. Working paper series n ${ }^{\circ} 64$, feb.

BACKHOUSE, Roger E. e LAIDLER, David. (2003). What was lost with IS-LM. Paper prepared for the HOPE conference "The IS-LM model: its rise, fall and strong persistence". Duke University, mimeo. BARRO, Robert J. (1993). Macroeconomics. $4^{\text {a }}$ ed. New York: John Wiley \& Sons.

BIANCHI, Ana M. (1992). "Hicks e a revolução invisível: notas sobre a contribuição metodológica de J.R. Hicks.” Revista Brasileira de Economia, vol. 46, n 1, pp. 131-148.

BLANCHARD, Olivier J. (1987) "Neoclassical Synthesis.” In: Eatwell, J., Milgate, M., E Newman, P. (eds.). The new palgrave: a dictionary of economics. London: Macmillan, 1987, pp. 634-636.

. (1992). "Novos-clássicos e novos keynesianos: a longa pausa.” Literatura Econômica, Rio de Janeiro, número especial, pp. 16-30.

. (1997). "Is there a core of usable macroeconomics?" American Economic Review (Papers and

Proceedings), vol. 87, $\mathrm{n}^{\circ}$ 2, pp. 244-246.

. (2000). "What do we know about macroeconomics that Fischer and Wicksell did not?" Quarterly Journal of Economics, vol. 115, $\mathrm{n}^{\circ}$ 4, pp. 1375-1411.

- (2001). Macroeconomia: teoria e política econômica. Rio de Janeiro: Campus, $2^{a}$ ed. BLANCHARD, Olivier J. e FISCHER, Stanley. (1989). Lectures in Macroeconomics. Cambridge: MIT Press. BLINDER, Alan S. (1986). "Keynes after Lucas.” Eastern Economic Journal, vol. 12, nº 3, pp. 209-216.

. (1988). "The fall and rise of keynesian economics." Economic Record, vol. 64, n 2, pp. 278294.

. (1997). "Is there a core of practical macroeconomics that we should all believe?" American Economic Review (Papers and Proceedings), vol. 87, n 2, pp. 240-243.

BORDO, Michael D. e SCHWARTZ, Anna J. (2003). IS-LM and Monetarism. Paper prepared for the HOPE conference "The IS-LM model: its rise, fall and strong persistence". Duke University, mimeo. BRAYTON, Flint; LEVIN, Andrew; TYRON, Ralph e WILLIAMS, John (1997). "The evolution of macro models at the Federal Reserve Board." Carnegie-Rochester Series on Public Policy, vol. 47, $\mathrm{n}^{\circ}$ 1, pp. 43-81. CHAMPERNOWNE, D. G. (1936). "Unemployment, basic and monetary: classical analysis and the keynesian." Review of Economic Studies, v. 3, n 1, pp. 201-16.

COLANDER, David. (2003). The strange persistence of the IS/LM model. Paper prepared for the HOPE conference "The IS-LM model: its rise, fall and strong persistence". Duke University, mimeo. COLANDER, David e KLAMER, Arjo (1990). The making of an economist. Boulder: Westview. COOLEY, Thomas J. (1995). Frontiers of Business Cycle Research. New Jersey: Princeton Univ. Press.

DANTHINE, Jean-Pierre (1997). "In search of a successor to IS-LM." Oxford Review of Economic Policy, vol. 13, $\mathrm{n}^{\circ}$ 3, pp. 135-144.

DARITY, William, Jr. e YOUNG, Warren (1995). "IS-LM: an inquest.” History of Political Economy, vol. $27, \mathrm{n}^{\circ} 1$, pp. 1-41.

DORNBUSCH, Rudiger e FISCHER, Stanley (1991). Macroeconomia. $5^{\text {a }}$ ed. São Paulo: Makron Books.

EASTERLY, William (1999). "The ghost of financing gap: testing the growth model used in international financial institutions.” Journal of Development Economics, vol. 60, n 2, pp. 423-438.

EICHENBAUM, Martin (1997). "Some practical thoughts on stabilization policy". American Economic Review (Papers and Proceedings), vol. 87, n 2, pp. 236-239.

FRANCO, Gustavo H. B. (1983). "Uma introdução ao artigo 'O Sr. Keynes e os clássicos: uma sugestão de interpretação’, de J. R. Hicks.” Literatura Econômica, vol. 5, nº 2, pp. 125-38.

FREY, Bruno S.; POMMEREHNE, Werner W.; SCHNEIDER, Friedrich e GILBERT, Guy (1984). "Consensus and dissension among economists: an empirical inquiry.” American Economic Review, vol. 74, n 5 , pp. 986-994.

GALÍ, Jordi (1992). "How well does the IS-LM fit postwar U.S. data?” Quarterly Journal of Economics, vol. $107, \mathrm{n}^{\circ} 2$, pp. $709-738$.

GOODFRIEND, Marvin e KING, Robert G. (1997). "The new neoclassical synthesis and the role of 
monetary policy.” In: Bernanke, Ben S. \& Rotemberg, Julio J. (eds.) NBER macroeconomics annual. Cambridge: MIT, pp. 231-283.

GORDON, Robert J. (1990). "What is new-keynesian economics?" Journal of Economic Literature, vol. $28, \mathrm{n}^{\circ} 3$, pp. $1115-1171$.

(1993). Macroeconomics. New York: Harper Collins.

HALL, Robert E. e TAYLOR, John B. (1989). Macroeconomia: teoria, desempenho e política. Rio de Janeiro: Campus.

HARCOURT, Geoffrey C. “Bastard keynesians.” In: Eatwell, J., Milgate, M., \& Newman, P. (eds.). The new palgrave: a dictionary of economics. London: Macmillan, pp. 924-928, 1987.

HARROD, R. F. (1937). "Mr. Keynes and traditional theory." Econometrica, vol. 5, n 1, pp. 74-86. HELLER, Cláudia. (2002). "Notas sobre as razões do sucesso do modelo IS-LM". Anais do VII Encontro Nacional de Economia Política, Curitiba, 24 p.

HICKS, J. R. (1937). “Mr. Keynes and the 'classics': a suggested interpretation.” Econometrica, vol. 5, n⿳亠丷厂 1, pp. 147-59.

. Value and Capital. (1939). Oxford: Clarendon Press.

."IS-LM: an explanation” (1980). Journal of Post Keynesian Economics, vol. 3, n 2, pp. 13954.

KEYNES, John M. (1936). A Teoria Geral do Emprego, dos Juros e da Moeda. São Paulo: Abril, 1982 (Coleção 'Os Economistas'). Primeira edição em inglês, 1936.

KING, R.G. (1993). "Will the new keynesian macroeconomics resurrect the IS-LM model?” Journal of Economic Perspectives, vol. 7, n 1 , pp. 67-82.

(2000). "The New IS-LM Model: Language, Logic, and Limits" Federal Reserve Bank of Richmond Economic Quarterly, vol. 83, $\mathrm{n}^{\circ}$ 3, pp. 45-102.

KLAMER, Arjo. (1988). Conversas com economistas: os novos economistas clássicos e seus opositores falam sobre a atual controvérsia em macroeconomia. São Paulo: USP.

(1989). "An accountant among economists: conversations with Sir John R. Hicks". Journal of Economic Perspectives, vol. 3, no 4, pp. 167-180.

KRUGMAN, Paul (1999a). “The world's smallest macroeconomic model.” (Disponível na Internet no endereço <http://web.mit.edu/krugman/www/MINIMAC.html>).

. (1999b). "There's something about macro." (Disponível na Internet no endereço <http://web. mit.edu/krugman/www/islm.html>).

LEIJONHUFVUD, Axel (1987). “IS-LM analysis”. In: Eatwell, J., Milgate, M. e Newman, P. (Eds.). The new palgrave: a dictionary of economics. London: Macmillan, pp. 302-307.

LIMA, Gilberto T. (1992). Em busca do tempo perdido: a recuperação pós-keynesiana da economia do emprego de Keynes. Rio de Janeiro: BNDES.

LJUNGQVIST, Lars e SARGENT, Thomas J. (2000). Recursive Macroeconomic Theory. Cambridge: MIT Press, 2000.

LOPES, Carlos M. (1992a). “Apresentação”. Revista Brasileira de Economia, vol. 46, nº 1, pp. 5-6. . (1992b). "IS-LM: mitos, controvérsias e enigmas.” Revista Brasileira de Economia, vol. 46, n 1 , p. 25-33.

LUCAS, Robert E., Jr. (1976). "Econometric policy evaluation: a critique.” Carnegie-Rochester Conference Series on Public Policy, vol. 1, pp. 19-46.

. (1980). "Methods and problems in business cycle theory." Journal of Money, Credit and Banking, vol. $12, \mathrm{n}^{\circ}$ 4, pp. 696-715.

LUCAS, Robert E. Jr. e SARGENT, Thomas J. (1979). “After keynesian macroeconomics.” Federal Reserve Bank of Minneapolis Quarterly Review, vol. 3, $\mathrm{n}^{\circ}$ 2, pp. 1-16.

MAGALHÃES, Matheus A. de. (2000). "Explicando os ciclos de negócios." Revista de Economia Aplicada, vol. 4, n 1, pp. 167-194.

MANKIW, N. G. (1990). “A quick refresher course in macroeconomics.” Journal of Economic Literature, vol. 28, $\mathrm{n}^{\circ} 4$, pp. 1145-60.

(1992). "The reincarnation of keynesian economics." European Economic Review, vol. 36, $\mathrm{n}^{\circ}$ 2, pp. 559-565.

(1995). Macroeconomia. Rio de Janeiro: LTR, 356 p. 
MANKIW, N.G. e ROMER, David H. (eds.). (1991a). New keynesian economics. Vol. 1: imperfect competition and sticky prices. Cambridge: MIT Press.

(1991b). New keynesian economics. Vol. 2: coordination failures and real rigidities. Cambridge: MIT Press.

MCCALLUM, Bennett T. e NELSON, Edward (1999). "An optimizing IS-LM specification for monetary policy and business cycle analysis." Journal of Money, Credit and Banking, vol. 31, $\mathrm{n}^{\circ}$ 2, pp. 296316.

MEADE, James R. (1937). “A simplified model of Mr. Keynes’ system.” Review of Economic Studies, vol. $4, \mathrm{n}^{\circ} 1$, pp. 98-107.

MODIGLIANI, Franco (1944). "Liquidity preference and the theory of interest and money." Econometrica, vol. $12, \mathrm{n}^{\circ} 1$, pp. $45-88$.

PATINKIN, D. (1990). "In Defense of IS-LM” Banca Nazionale del Lavoro Quarterly Review, 172, pp. 119-34.

POLICANO, Andrew J. (1985). "The current state of macroeconomics: a view from the textbooks." Journal of Monetary Economics, vol. 15, $\mathrm{n}^{\circ}$ 2, pp. 389-397.

ROMER, David H. (1996). Advanced Macroeconomics. New York: McGraw-Hill, 540 p. . (2000). "Keynesian macroeconomics without the LM curve". Journal of Economic Perspectives, vol. 14, n 2, spring, pp. 149-169.

SACHS, Jeffrey e LARRAIN, Felipe. (1995). Macroeconomia. São Paulo: Makron Books, 904 p. SAMUELSON, Paul A. (1955). Economics. New York: McGraw-Hill, $3^{\text {a }}$ ed.

SARGENT, Thomas J. (1987a). Dynamic Macroeconomic Theory. Cambridge: Harvard Univ. Press. . (1987b). Macroeconomic Theory. New York: Academic Press.

SOLOW, Robert M. (1984). "Mr. Hicks and the classics." Oxford Economic Papers, vol. 36, supplement, pp. 13-25.

(1997). "Is there a core of usable macroeconomics we should all believe in?" American Economic Review (Papers and Proceedings), vol. 87, $\mathrm{n}^{\circ}$ 2, p. 230-232.

STADLER, George W. (1994). "Real business cycles." Journal of Economic Literature, vol. 32, nº 4, pp. $1750-1783$.

SUMMERS, Lawrence H. (1991) “The scientific illusion in empirical macroeconomics." Scandinavian Journal of Economics, vol. 93, n 2, pp. 129-148.

TAYLOR, John B. (1997). "A core of practical macroeconomics.” American Economic Review (Papers and Proceedings), vol. 87, $\mathrm{n}^{\circ} 2$, pp. 233-235.

TOBIN, James (1986). “O futuro da economia keynesiana.” Pesquisa e Planejamento Econômico, vol. $16, \mathrm{n}^{\circ} 2$, pp. $237-250$.

UZABIAGA, Carlos (1998). Interview with Lawrence J. Christiano, mimeo.

VERCELLI, Alessandro (1999). "The evolution of IS-LM models: empirical evidence and theoretical pressupositions." Journal of Economic Methodology, vol. 6, n 2, pp. 199-219.

WOODFORD, Michael (1999). "Revolution and evolution in twentieth-century macroeconomics". Princeton University, mimeo. (Disponível na Internet no endereço http://www.princeton.edu/ woodford/). 